\title{
Noise Reduction in Medical Images using an Unbiased Non-local Means Method
}

\author{
Sushma $C^{1}$, Kavitha $\mathbf{G}^{2}$ \\ M.Tech Student, Department of Computer Science and Engineering, UBDTCE, Davangere, India ${ }^{1}$ \\ Assistant Professor, Department of Computer Science and Engineering, UBDTCE, Davangere, India ${ }^{2}$
}

\begin{abstract}
Medical ultrasound also known as diagnostic sonography or ultrasonogrophy is a diagnostic imaging technique based on the application of ultrasound. Since the speckle intensity has a signal dependent nature, filters based on the standard additive Gaussian noise model are inadequate. The main objective of the system is to remove the noise present in the image using UNLM Filter. The proposed unbiased NLM speckle filter based on Gamma statistics has mainly two phases. In the first phase the shape and scale parameters, $\rho$ and $\beta$, of the Gamma distribution are computed. In the second phase, an Unbiased Non-Local Means Method (UNLM) method was applied. Experiments were carried out on synthetic, B- mode US, CT scan, MRI images to validate the performance of the proposed method. Quantitative analysis based on PSNR with various noise levels shows that the proposed method is more effective in denoising.
\end{abstract}

Keywords: Denoising, ultrasound, MRI, CT scan images, NLM.

\section{INTRODUCTION}

Medical ultrasound also known as diagnostic sonography or ultrasonogrophy is a diagnostic imaging technique based on the application of ultrasound. It is used to see internal body structures such as tendons, muscles, joints, vessels and internal organs.

Basic filters were used to remove noise. But basic filters like median filters, order filters, non-linear filters will work only for some category of noise in the images. By applying these filters, there are chances of distorting the ROI where some important tumors may unable to identify. This leads to wrong analysis. Since the speckle intensity has a signal dependent nature, filters based on the standard additive Gaussian noise model are inadequate. Therefore, specific filters are required to suppress speckle without compromising important image features.

A plethora of despeckling methods have been developed for improving the quality of US images that can be performed either in the transform domain or in the spatial domain [5]. Loizou et al. in [3] conducted a comparative study of different US despeckling methods for carotid artery, and provided a Matlab toolbox for US image despeckling. The details are available in [6]. The Lee's filter [7], Frost's filter [8], and Kuan's filter [9] are the most widely discussed spatial adaptive filters to attenuate the speckle noise. These classical filters consider the speckle as multiplicative noise and described them mathematically using a Gaussian distributed noise model. Lopes et al. [10] proposed improved versions of the Lee's and Frost's filters by organizing the pixels in different classes in which precise processing is defined. Squeeze box filter (SBF) designed in [11,12] removes outliers at each iteration and smooths the random distributed pixel values to some confining value through adaptively computed mean. Recently, the RayleighMaximum-Likelihood (RML) filter in [13] was employed with the Rayleigh density model and the ML method was adapted for solving the estimation problem.

P V Sudeep in [1] presented an UNLM filter which removes speckle only from ultrasound images. They used a three parameter Gamma distribution function to fit the real US image and the ML estimation was adapted to find the two key parameters that control the filter performance. The proposed filter was scientifically validated by taking into consideration both real US and synthetic standardized images. We have extended this UNLM to work on different types of images like ultrasound, synthetic, CT scan and MRI images.

A new variant of NLM filter called unbiased NLM (UNLM) filter for Gamma distributed medical images in which we make the bias signal independent, thereby subtracting from the NLM filtered signal is proposed.

The main objective of the system is to remove the noise present in the image using UNLM Filter. Some tumors are like small dark dots in the images. These dots which represent tumors may be identified as noise and removed by the filter. This leads to wrong analysis by the doctors. Hence, our objective is to preserve such small dots in the images. 


\section{UNLM}

An unbiased NLM speckle filter based on Gamma statistics has been presented. We used a three parameter Gamma distribution function to fit the medical image and the ML estimation was adapted to find the two key parameters that control the filter performance.

The NL-Means filter is a non-linear, edge preserving filter that computes each output pixel as a weighted sum of input pixels. The set of input pixels contributing to one output pixel may stem from a large region in the input image, hence the term non-local.

A key feature of the NL-Means filter is that the weights are determined by the distance between small image patches. The NL-Means filter is a generalization of the bilateral filter, which considers distances between pairs of pixel values, instead of small patches, to compute filter weights.

An important observation is that, because the input signal is noisy, the measured squared distances are biased. Therefore, the original NL-Means filter subtracts the variance of the measured squared distances to cancel out the noise contribution from the patch distance.

Consider the discrete noisy image $\mathrm{U}=\mathrm{u}(\mathrm{y}) \mid \mathrm{y} \in \mathrm{R}^{\mathrm{N}}$, where $\mathrm{u}(\mathrm{y})$ corresponds to the noisy image value at pixel location $\mathrm{y}$. Let us assume that the search window size and similarity window size be $\left(\left(2 t_{1}+1\right) \times\left(2 t_{1}+1\right)\right)$ and $\left(\left(2 t_{2}+1\right) \times\left(2 t_{2}+1\right)\right)$, respectively. The NLM estimator for the filtered value at a location $r$ is defined as follows:

$$
\hat{u} N L M(u(r))=\sum_{\forall s \in \Omega} w(r, s) u(s)
$$

where $0 \leq \mathrm{w}(\mathrm{r}, \mathrm{s}) \leq 1, \sum_{\forall \mathrm{s} \in \Omega} \mathrm{w}(\mathrm{r}, \mathrm{s})=1$ and $\mathrm{s}$ represents each one of the pixel in the search window $\Omega$. The weight $\mathrm{w}(\mathrm{r}$, s) can be expressed as:

$$
w(r, s)=\frac{1}{Z(r)} e^{-\frac{d(r, s)}{h^{2}}}
$$

Where the decay parameter $\mathrm{h}$ controls the degree of smoothing and usually computed as the standard deviation of the noise. $\mathrm{Z}(\mathrm{r})$ is the normalizing constant and can be determined as:

$$
Z(r)=\sum_{\forall s \in \Omega} e^{-\frac{d(r, s)}{h^{2}}}
$$

Also, $d$ denotes a Gaussian weighted Euclidean distance of all the pixels in the search window. It is defined as:

$$
\mathrm{d}(\mathrm{r}, \mathrm{s})=\mathrm{G} \sigma|| \mathrm{Br}-\mathrm{Bs}||^{2} \mathrm{t} 2
$$

where $\mathrm{G}_{\sigma}$ is a normalized Gaussian weighting function with zero mean and $\sigma$ standard deviation.

We model the B-mode medical image with three parameter Gamma distribution. Then, we replace the third parameter $\Upsilon$ in order to convert the three parameter estimation problem to two parameter estimation problem. As a result, this improves the accuracy of estimation of $\rho$ and $\beta$. We can observe that the noise bias produced by the Gamma distribution is signal independent and the product $\rho \times \beta$ is nothing but the bias term. It directly represents the signal independent noise term. So, we modified the NLM filtered output to get the unbiased NLM output. For each pixel, the proposed NLM estimation can be defined as follows:

$$
\mathrm{NLMs}_{\text {peckle }}(\mathrm{U})=\max \left(\mathrm{NLM}(\mathrm{U})-\hat{\rho}_{\mathrm{ML}} \beta_{\mathrm{ML}}, 0\right)
$$

\section{EXPERIMENTAL RESULTS}

In order to validate the proposed methodology and to do a visual check of the performance of various filters, a new experiment is carried out on phantom and real ultrasound data sets. Results obtained are shown in figures 1 and 2 respectively. Figures 3 and 4 show the results obtained from CT scan and MRI images. Experiments were carried out on synthetic, B-mode US, CT scan, MRI images to validate the performance of the proposed method. The obtained PSNR values of these images are shown in the tables. 


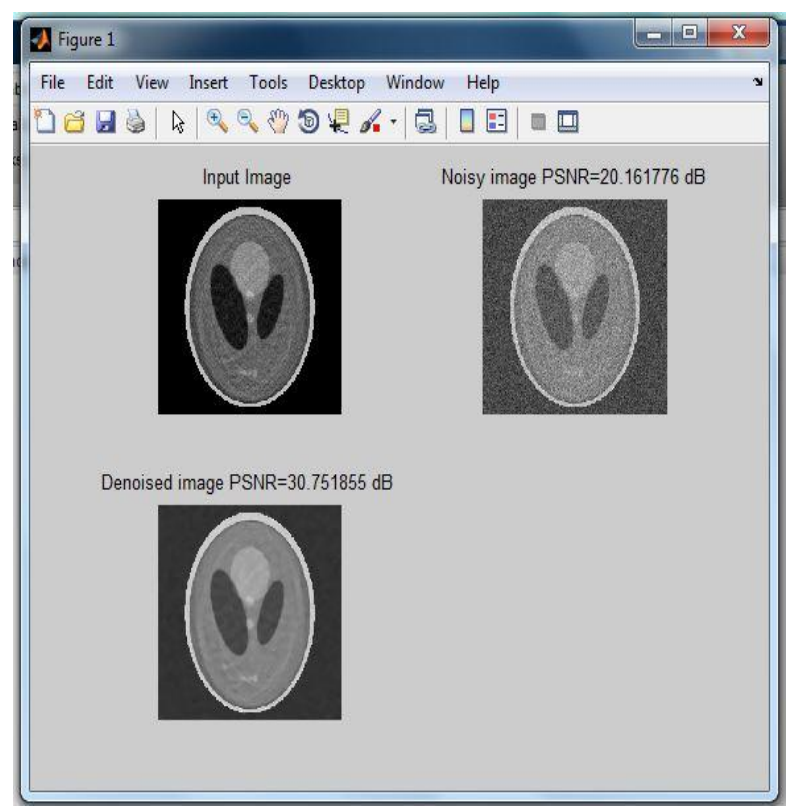

Figure. 1. Results obtained with proposed filter applied to the Phantom image corrupted with Gaussian noise.

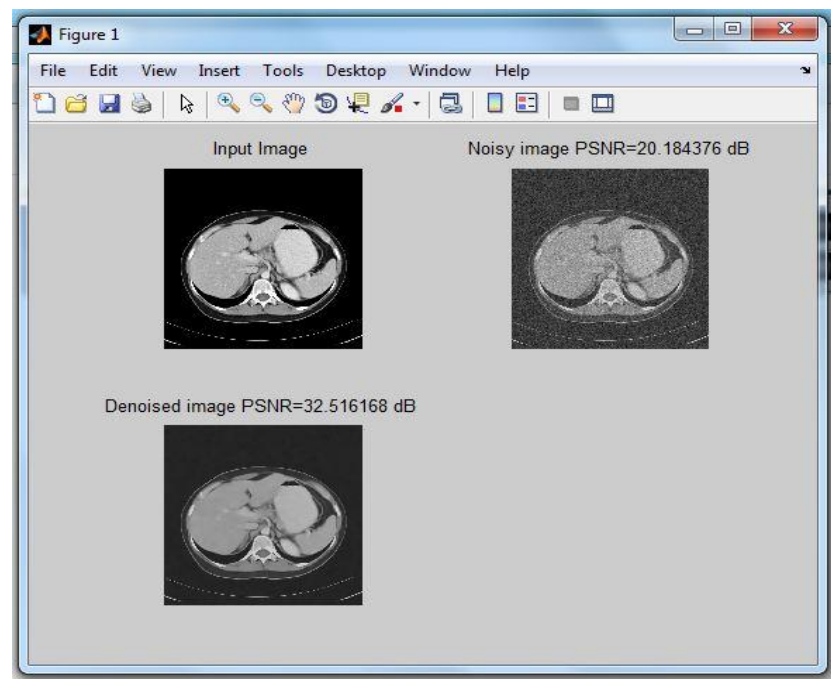

Figure 2. Results obtained with proposed UNLM filter applied to the real US image

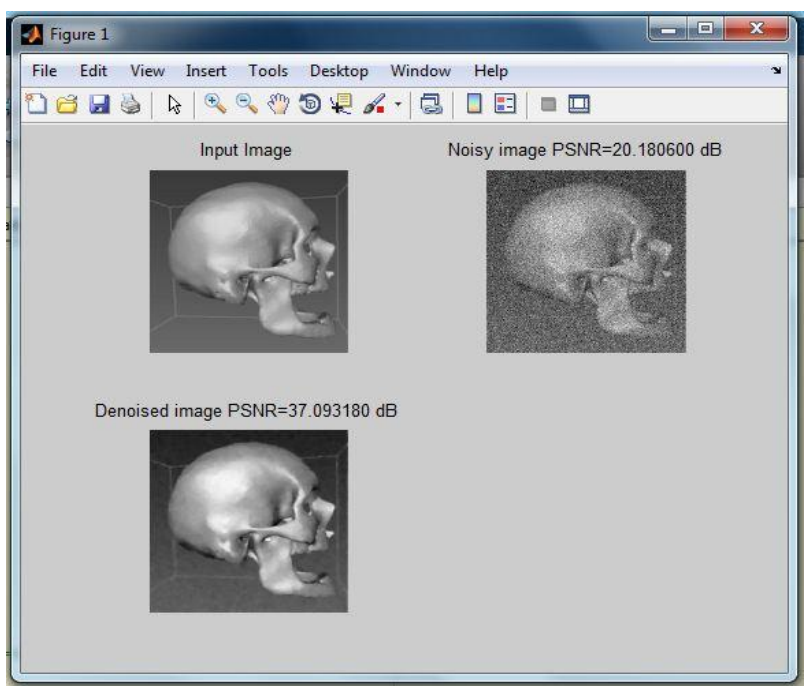

Figure 3. Results obtained with proposed UNLM filter applied to the CT scan image 


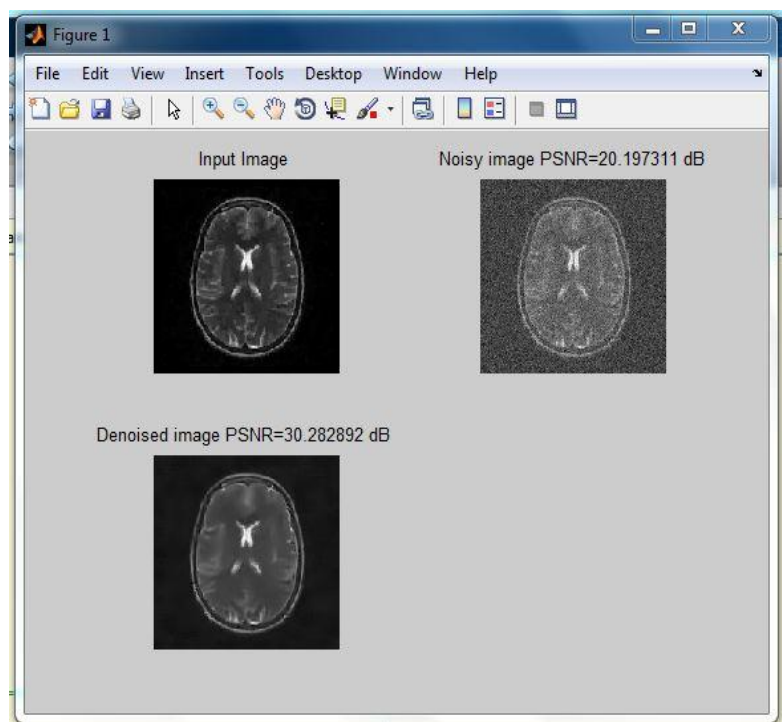

Figure 4. Results obtained with proposed UNLM filter applied to the MRI image

Table 1. PSNR values of ultrasound images

\begin{tabular}{|l|l|l|}
\hline Input Image & Noisy Image PSNR value-db & Denoised Image PSNR value-db \\
\hline us_1 & 20.194643 & 36.894507 \\
\hline us_2 & 20.164632 & 36.552914 \\
\hline us_3 & 20.173343 & 36.653685 \\
\hline us_4 & 20.182302 & 36.494665 \\
\hline Average & 20.17873 & 36.648942 \\
\hline
\end{tabular}

Table 2. PSNR values of CT scan images

\begin{tabular}{|l|l|l|}
\hline Input Image & Noisy Image PSNR value-db & Denoised Image PSNR value-db \\
\hline CT_1 & 20.180600 & 37.093180 \\
\hline CT_2 & 18.571317 & 36.038119 \\
\hline CT_3 & 18.113343 & 30.153685 \\
\hline CT_4 & 18.112102 & 30.194665 \\
\hline Average & 18.744340 & 33.369912 \\
\hline
\end{tabular}

Table 3. PSNR values of phantom images

\begin{tabular}{|l|l|l|}
\hline Input Image & Noisy Image PSNR value-db & Denoised Image PSNR value-db \\
\hline Phantom_1 & 20.111776 & 30.151855 \\
\hline Phantom_2 & 20.124632 & 30.141855 \\
\hline Phantom_3 & 20.113343 & 30.153685 \\
\hline Phantom_4 & 20.112102 & 30.194665 \\
\hline Average & 20.115463 & 30.16051 \\
\hline
\end{tabular}

Table 4. PSNR values of MRI images

\begin{tabular}{|l|l|l|}
\hline Input Image & Noisy Image PSNR value-db & Denoised Image PSNR value -db \\
\hline MRI_1 & 20.197311 & 30.282892 \\
\hline MRI_2 & 20.187077 & 28.709250 \\
\hline MRI_3 & 20.113343 & 30.153685 \\
\hline MRI_4 & 20.112102 & 28.194665 \\
\hline Average & 20.152458 & 29.335123 \\
\hline
\end{tabular}

The tables show PSNR values of ultrasound, CT scan, MRI, phantom images. By these tables, we can conclude that the proposed UNLM filter works for different types of images and its best for ultrasound images. 


\section{CONCLUSION}

The proposed unbiased NLM speckle filter based on Gamma statistics has mainly two phases. In the first phase the shape and scale parameters, $\rho$ and $\beta$, of the Gamma distribution are computed. In the second phase, an unbiased NLM method was applied. Experiments were carried out on synthetic, B-mode US, CT scan, MRI images to validate the performance of the proposed method. Quantitative analysis based on PSNR with various noise levels shows that the proposed method is more effective in denoising. Moreover visual analysis indicated that the image denoised by the proposed method is superior.

\section{REFERENCES}

[1] P.V. Sudeep, Luca Saba, Jeny Rajan, Jasjit Suri, Speckle Reduction in Medical Ultrasound Images using an Unbiased Non-Local Means Method, Elsevier, 2016.

[2] T. Araki, N. Ikeda, D. Shukla, N.D. Londhe, V.K. Shrivastava, S.K. Banchhor, L.Saba, A. Nicolaides, S. Shafique, J.R. Laird, et al., A new method for IVUS-based coronary artery disease risk stratification: a link between coronary \& carotid ultrasound plaque burdens, Comput. Methods Progr. Biomed. 124 (2015)161-179.

[3] U.R. Acharya, S.V. Sree, S. Kulshreshtha, F. Molinari, J.E.W. Koh, L. Saba, J.S.Suri, GyneScan: an improved online paradigm for screening of ovarian cancervia tissue characterization, Technol. Cancer Res. Treat. 13 (6) (2014) 529-539.

[4] C.P. Loizou, C.S. Pattichis, C.I. Christodoulou, R.S. Istepanian, M. Pantziaris, A.Nicolaides, Comparative evaluation of despeckle filtering in ultrasound imaging of the carotid artery, IEEE Trans. Ultrason. Ferroelectr. Freq. Control52 (10) (2005) 1653-1669.

[5] A.A. Mahmoud, S.E. Rabaie, T. Taha, O. Zahran, F.A. El-Samie, W. Al-Nauimy, Comparative study of different denoising filters for speckle noise reduction in ultrasonic B mode images, IJIGSP 5 (2) (2013) 1-8.

[6] C.P. Loizou, C. Theofanous, M. Pantziaris, T. Kasparis, Despeckle filtering software toolbox for ultrasound imaging of the common carotid artery, Comput. Methods Progr. Biomed. 114 (1) (2014) 109-124.

[7] J.-S. Lee, Digital image enhancement and noise filtering by use of local statistics, IEEE Trans. Pattern Anal. Mach. Intell. 2 (2) (1980) 165168 .

[8] V. Frost, J. Stiles, K. Shanmugan, J. Holtzman, A model for radar images and its application to adaptive digital filtering of multiplicative noise, IEEE Trans.Pattern Anal. Mach. Intell. 4 (2) (1982) 157-166.

[9] D. Kuan, A. Sawchuk, T. Strand, P. Chavel, Adaptive noise smoothing filter for images with signal-dependent noise, IEEE Trans. Pattern Anal. Mach. Intell. 7 (2) (1985) 165-177.

[10] A. Lopes, R. Touzi, E. Nezry, Adaptive speckle filters and scene heterogeneity, IEEE Trans. Geosci. Remote Sens. 28 (1990) $992-1000$.

[11] P. Tay, S. Acton, J. Hossack, A stochastic approach to ultrasound despeckling, in: 3rd IEEE International Symposium on Biomedical Imaging: Nano to Macro, 2006, 2006, pp. 221-224.

[12] P. Tay, S. Acton, J. Hossack, Ultrasound despeckling using an adaptive window stochastic approach, in: 2006 IEEE International Conference on Image Processing, 2006, pp. 2549-2552.

[13] T. Aysal, K. Barner, Rayleigh-maximum-likelihood filtering for speckle reduction of ultrasound images, IEEE Trans. Med. Imaging 26 (5) (2007) 712-727. 